\section{Critical Potentials of the Vapours of Mercuric Halides.}

STUDying the losses of energy suffered by slow electrons in mercury vapour by the methods of Lenard (velocity distribution) and Franck and Hertz, we came across a rather sensitive arrangement for detecting the inelastic collisions of smaller probability (V. I. Pavlov, Jour. Russ. Phys.-Chem. Soc., Phys. Sect., v. 58, p. 369, I926). Applying this combined method to the investigation of the vapours of mercuric halides, we found for mercuric chloride at least four values for critical potentials: I.6 v., $2 \cdot 7$ v., 3.9 v., and $6.6 \mathrm{v}$.; for mercuric bromide: $\mathrm{I} \cdot 5 \mathrm{v}$., $2.9 \mathrm{v}$., 6.6 v., and 8.9 v.; for mercuric iodide: $\mathrm{I} \cdot 4 \mathrm{v}$. , 2.7 v., 6.2 v., and 7.8 v. The same method for iodine yielded the values: $I \cdot 2$ v., $2 \cdot 3$ V., 3.8 v., $5 . I$ V., and 7.5 v. Some of these data seem to be in agreement with the observed absorption by the vapours of light of corresponding quanta.

It may be hoped that the results obtained will lead to the building up of a system of energy levels of the molecules investigated and will give some knowledge of the various possible ways of dissociation with excitation.

V. I. PAVLOV.

A. I. LEIPUNSKY.

Physical and Technical Laboratory, Leningrad, November I 8 .

\section{Optical Excitation of the Vapours of Mercuric Halides.}

In the course of an investigation on the optical excitation of salt vapours I have found that vapours of mercuric chloride, mercuric bromide, and mercuric iodide of low density, when illuminated by the spark, give a bright visible fluorescence; this is green for mercuric chloride, blue for mercuric bromide, and violet for mercuric iodide. The fluorescence consists of a system of bands with a somewhat abrupt maximum of intensity at respectively $5600 \AA$.U., $5000 \AA$.U., and $4400 \AA . U$., and there is a continuous falling of intensity down to about 4000 A.U.

The lines which stimulate these bands are strictly confined to the following rather narrow limits: I90oI 850 for mercuric chloride, 2100-1900 for mercuric bromide, and 2200-2Ioo for mercuric iodide. A detailed analysis of the structure of these bands, together with further experimental work, will give; I hope, some information upon the underlying mechanism,

Optical Institute, Leningrad.

$$
\text { A. TERENIN. }
$$

\section{Sir William Grookes and Spiritualism.}

IN the leading article in NATURE of November 20 , it is stated that Sir William Crookes closed the spiritualistic chapter for the rest of his life for reasons which, owing to the deliberate destruction of the necessary documents, it is impossible to gauge.

I am in a position to throw light on this matter, and as what I have to say is purely historical, and does not touch on the marvellous, it will perhaps not be considered to fall under the ban of the editorial closure.

Sir William Crookes, on a visit to my parents in August I894, engaged in conversation with my grandmother, the second Lady Rayleigh, on this subject. I listened with attention. The conversation was substantially as follows :

Lady $R$. Have you had any recent spiritualistic experiences?

$$
\text { NO. } 2980 \text {, VOL. II } 8]
$$

Crookes. No, unfortunately not. The difficulty is that I have not been able to get into touch with any suitable medium lately.

Lady $R$. Well, that is unfortunate; but after the wonderful things that you have seen perhaps you may fairly be satisfied.

Crookes. I do not know that. When one has had a good dinner one day, one none the less wants another the next day.

The last sentence is quite clear in my recollection.

Terling Place, Chelmsford, Essex, November 26 .

\section{Behaviour of Silicic Acid Gel during the Drying-up Process.}

IN a note published in the Proceedings of the Indian Association for the Cultivation of Science (vol. 9, Part 4, p. 328, June I926), I described an observation made by me more than two years ago (in April 1924), namely, that when silicic acid gel is treated with a concentrated solution of potassium chloride and is then allowed to dry up, the salt exudes from the surface of the gel in the form of thin white, glistening fibres. I attributed this to the pressure that is exerted upon the capillaries of the gel, as the gel continually loses water and experiences contraction. A photograph of the growth was also published there. It was further pointed out that an X-ray examination of those fibres of potassium chloride might reveal their true nature. As indicated before, this phenomenon seems to be very general, and should therefore be observable with various gels and salts.

The note referred to was written long ago, but its publication was delayed, as I intended to study the phenomenon further.

In a paper on the " Structure of Silicic Acid Gels," communicated by Messrs. Fells and Firth to the Proc. Roy. Soc. (I I 2 A, Sept. I926, p. 468), the authors describe interesting experiments on the same phenomenon. Apparently my previous note was not noticed by them.

Ramsay Chemical Laboratories,

K. Krishnamurti. University College, London.

\section{The Reaction to Flea Bites.}

Prof. E. A. Boycote, in Nature of October 23. says: "Some persons who are extensively flea bitten seem to become immune." This is, in my case, true of other insect bites. In January I 885 I suffered the most terrible agony from bites of Australian sandflies (I do not know the specific name). Some seven years afterwards I was again in a sand-fly-infested neighbourhood and was completely immune from effects, and was so ever since.

In January I906 I spent four or five days fishing in New Zealand, where I suffered misery from the bites of the New Zealand "sand-fly." (This is quite a different insect from its Australian namesake.) Returning again in January 1907 I suffered almost as much. I visited the same spot for four or five years later, and, though attacked as much as before, never felt any effects.

Similarly the mosquito, which was once a terror to me, now does no more than leave a painless mark. It is perhaps curious that the 'gruelling 'I had from the Australian species has conferred immunity in Europe.

Oxford, November 2. 